\title{
THE NEED FOR THE SOCIAL DISTANCING ON THE CROWD MANAGEMENT OF MAJOR EVENTS DURING CORONAVIRUS: A CASE OF THE ISLAMIC PILGRIM (HAJJ)
}

\author{
Dr. Raed Nayif Alahmadi \\ Civil Engineering Department, \\ Albaha University, Saudi Arabia
}

\begin{abstract}
Crowds are a feature of large cities, occurring not only at mass gatherings but also at routine events such as the journey to work, schools, churches, mosques, campaign events, carnivals and lots more. Bringing people together has many positive social and economic benefits. Some environments for mass gatherings are so crowded that the risk of a disaster is ever present. The Olympic Games, the Hajj, the Christian pilgrimage and other major sporting, religious and cultural events are some example of such a crowded environment. On March 11, 2020, the World Health Organization declared COVID-19 a pandemic. Events involving large crowds have been cancelled or postponed worldwide; access to public buildings has been restricted as different mitigation measures have been adopted around the world to decrease physical interactions among people during the COVID-19 pandemic. Strategies for containing an emerging infectious disease outbreak must be non-pharmaceutical when drugs or vaccines for the pathogen do not yet exist or are unavailable. The success of these non-pharmaceutical strategies will depend on not only the effectiveness of isolation measures but also the epidemiological characteristics of the infection. Infectious diseases are commonly controlled by minimizing contact between infectious and susceptible individuals. Personal measures to reduce potentially infectious contacts are sometimes referred to as 'social distancing'. It has been suggested that policies encouraging social distancing may be effective against pandemic influenza (Bell et al. 2006; Glass et al. 2006). An examination of levels of social distancing actually achieved during previous epidemics can provide useful guidance as to the effectiveness of social distancing interventions during future influenza pandemics.
\end{abstract}

Keywords: Crowds, Mass gathering; COVID -19, Pandemic, Social Distancing, Hajj, Pilgrim

Cite this Article: Dr. Raed Nayif Alahmadi, The Need for the Social Distancing on the Crowd Management of Major Events During Coronavirus: A Case of the Islamic Pilgrim (HAJJ), International Journal of Civil Engineering and Technology, 11(6), 2020, pp. 111-120.

https://iaeme.com/Home/issue/IJCIET?Volume $=11 \&$ Issue $=6$ 


\section{INTRODUCTION}

Crowds are a feature of large cities, occurring not only at mass gatherings but also at routine events such as the journey to work, schools, churches, mosques, campaign events, carnivals and lots more. Bringing people together has many positive social and economic benefits. Financing and organizing cultural events linked to history and traditions of a community can lead to its overall development, due not only to the direct and to the indirect economic impact the tourist flow produces in the short-term. There is also the long term promotion and the creation of social ties and sets the conditions for a sustainable economic development of the territory. However, crowds or mass gatherings also have several negative outcomes. One of such negative outcomes associated with mass gathering is the development and spread of some diseases among which are respiratory epidemics. Frequent interactions between people whose physical contact increase non-linearly with the density of individuals in any particular place results in transmission of contagious diseases to a large population in the shortest time. During any mass gathering, environmental and public health planning includes protecting the health and wellbeing of participants, staff, and spectators from infections, other illnesses, and injuries related to improper management of food, water, waste, land, and traffic. Health authorities need to consider basic human needs, including potable water, sufficient public toilets, adequate refrigeration for perishable foodstuff s, recognized and approved suppliers of bulk foodstuff $s$ to the food providers at the site, sufficient capabilities for the disposal of liquid and solid waste, appropriate storage and removal of liquid waste, and control of rodents and insects that affect health.

Some environments for mass gatherings are so crowded that the risk of a disaster is ever present. The Olympic Games, the Hajj, the Christian pilgrimage and other major sporting, religious and cultural events are Some example of such a crowded environment. Meanwhile, Infectious diseases are commonly controlled by minimizing contact between infectious and susceptible individuals. Personal measures to reduce potentially infectious contacts are sometimes referred to as 'social distancing'. It has been suggested that policies encouraging social distancing may be effective against pandemic influenza (Bell et al. 2006; Glass et al. 2006). An examination of levels of social distancing actually achieved during previous epidemics can provide useful guidance as to the effectiveness of social distancing interventions during future influenza pandemics.

To this end, this research has chosen the annual Hajj pilgrimage in Saudi Arabia as major religious event for this study considering its historic importance and previous efforts of the organizers to deal with issues of diseases out breaks in the past editions of the annual religious event.

\section{THEORETICAL FRAMEWORK}

Crowd management research has been going on since at least the 1890s when Gustave Le Bon studied the psychology of crowds by observing how they formed. Crowd behaviour is generic and Isaac Newton generalized its dynamics when referring to the "madness of crowds" during the financial crisis of the South Sea Bubble in the early 18th century. However, about 40 years ago, quantitative methods started to be used for crowd research, based on experiments under controlled conditions, to measure the effect of architectural configurations in buildings and streets on the flow of people, and study the video recordings of crowds.

Crowds or Mass Gatherings are defined by the World Health Organization (WHO) as occasions whether planned or spontaneous that attracts enough people to strain the planning and response resources of the community, city or nation hosting the event (World Health Organization, 2008). 
A classic tension currently exists in public health practice between efforts to ensure the health and safety of communities while simultaneously respecting the liberties of individuals within those communities (Gostin 2000). Nowhere is this tension more apparent than in pandemic planning and response. As we have seen in the current response to the COVID 19 outbreak, public health officials implement a variety of social distancing measures (e.g., school and business closures, quarantine) in communities to contain contagion. In doing so, these measures limit personal freedoms and may create serious ethical challenges for institutions and larger communities (Gostin 2006; Schuklenk and Gartland 2006; Thompson et al. 2006; Zhang et al. 2006; Kotalik 2005).

The novel coronavirus (COVID-19) pandemic is considered the greatest public health threat since the 1918 Influenza Pandemic that infected one-third of the world's population and killed at least 50 million people. COVID-19 cases and fatalities are growing exponentially and there is much uncertainty about its ultimate impacts globally. Perhaps as unsettling as these estimates is the uncertainty around the health impacts that are wracking societies with fear.

On March 11, 2020, the World Health Organization declared COVID-19 a pandemic. Events involving large crowds have been cancelled or postponed worldwide, access to public buildings has been restricted as different mitigation measures have been adopted around the world to decrease physical interactions among people during the COVID-19 pandemic (Anderson et al., 2020). The measures to face pandemics adopted by policy makers ranged from compulsory lockdowns to recommendations on social distancing (also called physical distancing). In the absence of vaccines, countries around the world are implementing various forms of "social distancing" as a policy to slow the virus' spread. This social distancing takes many forms but, at its core, its aim is to keep people apart from each other by confining them to their homes in order to reduce contact rates. The impacts of social distancing are evident in the data from China, especially when compared with Italy which implemented social distancing policies more slowly and sporadically. At the same time, the economics costs are clear in the Chinese and Italian data, and in the US Goldman Sachs is projecting quarter on quarter annualized growth rates of $-6 \%$ in Q1 and -24\% in Q2 (Hatzius et al. 2020). Further, historically unprecedented US unemployment claims have begun to arrive and the near term outlook for the job market is grim (Hatzius et al. 2020). The demonstrated benefits in China (as well as South Korea and Singapore) and the sharp and large economic costs naturally raise critical questions about whether social distancing is worth it (Hilsenrath and Armour 2020; Bender and Ballhaus 2020; Thunstrom et al. 2020).

One key aspect sticks out in the current situation, i.e. the inconsistency in the adopted measures is evident. For example, social distancing recommendations are diverse in different countries and rapidly change over time, e.g., 1 person/4 $\mathrm{m} 2$ (Australia), $1 \mathrm{~m}$ (Philippines, Qatar) or 2 m outside home (Canada, UAE, UK, South Korea), or 1-2 m (New Zealand, Italy) (Italian Government, 2020; Korean Ministry of Economy and Finance, 2020; Movement Strategies, 2020). This raises questions on their implications on space usage (Honey-Roses et al., 2020), how policy makers have grounded their decisions and what type of models are currently available to support these decisions. Moreover, current data do not fully support the 1-2 m rule for spatial separation assumed for travelling of large droplets (Bahl et al., 2020) which has been issued in the airborne precautions for the COVID-19 pandemic in the guidelines by the World Health Organization (WHO, 2020). This issue highlights the need for flexible models able to use different assumptions on disease transmission mechanisms.

In recent times, what decision makers have used in taking their decisions is mostly based on macroscopic epidemiological models, among which the most used are different variations of the SIR model which is a tool that estimates the number of people infected in given 
conditions over time. The SIR model divides the population into three types, namely (1) susceptible, S; (2) infectious, I; and (3) recovered/removed, R. The SIR model is based on earlier analytical approaches developed to study disease spread back in the 1920ies (Kermack $\&$ McKendrick, 1927). The current field of mathematical epidemiology adopts a set of differential equations which generally considers also a (4) exposed class, E, to create the socalled SEIR model (Anderson et al., 1992). Several other macroscopic epidemiological models have been developed and used, including stochastic transmission models (Kucharski et al., 2020) and mean-field epidemiological models (Giordano et al., 2020).

While the use of macroscopic epidemiological models provides the great benefit to be applicable at large scales and give vital information to decision makers as they yield predictions of the spread of disease. Nevertheless, their great limitation is that they may not fully consider the mobility patterns of individuals and the heterogeneity in their interactions.

Strategies for containing an emerging infectious disease outbreak must be nonpharmaceutical when drugs or vaccines for the pathogen do not yet exist or are unavailable. The success of these non-pharmaceutical strategies will depend on not only the effectiveness of isolation measures but also the epidemiological characteristics of the infection. However, there is currently no systematic framework to assess the relationship between different containment strategies and the natural history and epidemiological dynamics of the pathogen. (Peak et al., 2017).

The focus of this research is specifically on the impact of Social Distancing as one of the measures of curtailing the pandemic, while using the annual Hajj to Makkah in Saudi Arabia as the crowd and religious event. Although precautionary measures have been taken in Saudi Arabia to slow down the spread of COVID-19, such as locking down the most affected cities, practicing social distancing, and applying the infection-control precautions, it is the believe of the researcher that further exposition to other works of similar concern will add to the body of knowledge on the impact of Social Distancing on a historical event like the annual Hajj pilgrimage at the holy land in Makkah. Forecasting the COVID-19 progression in the city of Makkah will also help the policymakers decide if the Hajj will be able to operate this year.

\section{METHODOLOGIES \& RESULTS}

The research relied on case studies and literature review on mass gatherings and the application of medicines during pandemics and epidemics including crowd management and pandemic. The review was further extended to include media reports and published articles and reports about Hajj operations in Saudi Arabia.

There is an increasing recognition that our well-being is shaped by social factors such as our social relationships Various processes have been posited to explain this relationship. Some of these concern the beliefs and practices of particular groups. Others concern the more general features of group life. For example, being religious has a positive impact on wellbeing. In part, this is because religious beliefs provide cognitive schemas or encourage meditative practices relevant to the appraisal of life events and coping. In part, it is also because of one's participation in a congregation and the fact that one acts alongside others. In similar vein, research on work groups operating in stressful environments shows that the more one feels a part of the team, the better one's well-being and evidence from studies of the elderly living in residential homes reveals that a range of activities (from drinking water to avoid dehydration to reminiscing about the past) bring about benefits because they are done in groups. The underlying psychological process that link group membership with well-being is argued to derive from a sense of shared identity ('we-ness') that develops in groups. This leads people to experience mutual trust, respect and cooperation. It can also lead people to 
expect support from their fellow group members and develop greater resilience. Building upon such insights it has been argued that since we spend most of our time in the company of others, we should study health in group settings, (Tewari et al., 2012).

The Hajj is the largest annual gathering in the world, and it is a very important event for every Muslim. Makkah annually receives three million pilgrims who perform Hajj. (Mujallad \& khoj, 2020). At the Hajj, accommodation ranges from the most basic to the most sophisticated, but most pilgrims have to share public facilities and live in semipermanent tents. Inadequate storage, cooking, or transportation, lack of refrigeration, and lack of proper food handling can contribute to the pilgrim's risk of disease. With an attraction of about 3 million pilgrims during 1 week, It is very crowded, with millions of pilgrims undertaking their religious duties within strict constraints in terms of space and time; this rigour and strictness have led to a series of large crowd disasters over several years, thus putting pressure on the authorities. In the past few years, efforts have increased to solve this difficulty by scientific means, use of crowd simulation models, assessment of the best ways of grouping and scheduling pilgrims, crowd management and control, luggage management, video monitoring, and changes in the construction of the transport system for the event. However during the past 20 years, more advanced quantitative techniques have become increasingly popular because of advances and reduced costs of computation. New methods for data capture implement fi ne spatial scales such as those used in global positioning system (GPS) technologies. These techniques include the simulation of pedestrian flows, automated computer vision, and new methods for modeling navigation and route finding.

The other tools we have at hand are isolation and quarantine, social distancing and community containment. All these tools are currently being employed at an unprecedented massive scale in China. For a clearer understanding here is the definition of these tools, with explanation of how they are being used to control the novel coronavirus, and elaborate on the benefits and challenges (Wilder-Smith \& Freedman, 2020).

'Isolation' is the separation of ill persons with contagious diseases from non-infected persons to protect non-infected persons, and usually occurs in hospital settings. An isolation room could also be equipped with negative pressure to reduce transmission via aerosols, but for large droplets like for SARS CoV, control was achieved without negative pressure rooms. Isolation of patients is particularly effective in interrupting transmission if early detection is possible before overt viral shedding. Given that influenza patients can already transmit before clinical symptoms set it, isolation is often too late to be sufficiently effective to halt transmission and control an influenza pandemic. However, for SARS CoV the incubation time is longer than for influenza (about 5 versus 2 days), and viral shedding was highest once the patient is truly sick. A longer incubation time allows for more time to identify cases and put them into isolation. The incubation time of the 2019-nCoV also has a median of 5 days, however, at this stage, it remains unknown when viral shedding and transmissibility peaks and how frequently pre-symptomatic cases result in secondary cases.

'Quarantine' is one of the oldest and most effective tools of controlling communicable disease outbreaks. This public health practice was used widely in fourteenth century Italy, when ships arriving at the Venice port from plague-infected ports had to anchor and wait for 40 days (in Italian: quaranta for 40) before disembarking their surviving passengers. Forty days provided ample time for the incubation time to be completed so that yet asymptomatic cases became symptomatic and could therefore be identified. Quarantine was implemented successfully as an effective measure during the SARS epidemic in 2003. It is also an important component of pandemic influenza plans. Quarantine means the movement restriction of persons who are presumed to have been exposed to a contagious disease but are not ill either because they did not become infected or because they are still in the incubation 
period. Quarantine may be applied at the individual or group level and usually involves restriction to the home or a designated facility. Quarantine may be voluntary or mandatory. During quarantine, all individuals should be monitored for the occurrence of any symptoms. If such symptoms occur, they must be immediately isolated in a designated center familiar with treating severe respiratory illness. Quarantining is most successful in settings where detection of cases is prompt; contacts can be listed and traced within a short time frame with prompt issuance of quarantine with voluntary compliance to this issuance.

'Social distancing' is designed to reduce interactions between people in a broader community, in which individuals may be infectious but have not yet been identified hence not yet isolated. As diseases transmitted by respiratory droplets require certain proximity of people, social distancing of persons will reduce transmission. Social distancing is particularly useful in settings where community transmission is believed to have occurred, but where the linkages between cases is unclear, and where restrictions placed only on persons known to have been exposed is considered insufficient to prevent further transmission. Examples for social distancing include closure of schools or office buildings and suspension of public markets, and cancellation of gatherings (Wilder-Smith \& Freedman, 2020). This last control tool as has been defined was the focus of this research paper, particularly its impact within the context of a religious event like the Hajj.

\section{DISCUSSION AND INTERPRETATION OF RESULTS OBTAINED}

A critical question in pandemic planning is the role non-pharmaceutical interventions might play in delaying the temporal effects of a pandemic, reducing the overall and peak attack rate, and reducing the number of cumulative deaths. Such measures could potentially provide valuable time for pandemic-strain vaccine and antiviral medication production and distribution. Optimally, appropriate implementation of non-pharmaceutical interventions would decrease the burden on health care services and critical infrastructure. (Markel et al., 2007). As has been presented by (Wilder-Smith \& Freedman, 2020), a major tool within the context of non-pharmaceutical intervention is the use social distancing. The question is how effective can social distancing be as an interventionist tool?

(Bootsma \& Ferguson 2007; Hatchett et al. 2007) has postulated that the infectiousness of a disease is characterized by the basic reproduction number (R0), which for our purposes is the expected number of infectious contacts per infective when there are no pharmaceutical or behavioural interventions in place and every individual is equally susceptible. More sophisticated definitions are required where individuals have substantially different risks of infection; the methods described by Diekmann \& Heesterbeek (2000) are useful in defining and calculating R0 when contact structures and other kinds of heterogeneity are important. In practice, when an epidemic occurs, the effective reproduction number $(\mathrm{R})$ differs from $\mathrm{R} 0$ due to the deployment of interventions, the build-up of herd immunity and possibly pre-existing immunity. (Caley et al., 2008). The benefit arising from interventions that additionally decrease $\mathrm{R}$, beyond that expected based on herd immunity alone may differ depending on the magnitude of the decrease and its timing.

Because millions of pilgrims undertake their religious ritual within strict constraints in term of space and time; this rigour and strictness have led to a series of large crowd disasters over several years, thus putting pressure on the authorities. In the past few years, the government of Saudi Arabia has put an enormous effort to solve this difficulty using state of the art innovative scientific means. The use of crowd simulation models, assessment of the best ways of grouping and scheduling pilgrims, crowd management and control engineering technologies, luggage management, video monitoring, and changes in the construction of the transport system for the event. Although simulation studies were conducted on the basis of a 
wide range of assumptions, most of these studies suggested that isolation would reduce transmission, including reducing the epidemic size and delaying the epidemic peak. However, Fraser et al. (11) discussed the difficulty in controlling influenza transmission, even with high level of isolation combined with contact tracing and quarantine, because of the potentially high proportion of influenza transmission that occurs from mild or asymptomatic infections. (Fong et al., 2020).

(Federal Reserve Bank of Richmond \& Hornstein, 2020) has this to say about social distancing "Policy interventions such as social distancing and quarantining known infected individuals are modeled through their impact on the flow of new infections. As in the basic SIR model the flow of new infections is proportional to the product of susceptible individuals and infectious individuals, but quarantine can reduce the number of infected individuals that can meet the susceptible population. We assume that symptomatic individuals are always known, and that tracing and random testing can identify some of the exposed and asymptomatic individuals."

A further recent research on the impact of social distancing (Fong et al., 2020) has this lengthy report to buttress their findings on the subject: we note that the particular benefits estimates are only as reliable as Ferguson et al.'s projections on COVID-19's spread and health risks. The method can be used with any set of projections, so as more information arrives and research advances, this approach can be applied to other projections and to infer the benefits of alternative policy responses. Our emphasis is on Ferguson et al.'s "no policy" and mitigation social distancing scenarios. In the no policy scenario, there is uncontrolled growth of the coronavirus pandemic that leads to an $81 \%$ infection rate in the United States by October 1 and 2.2 million deaths. As a basis of comparison, in late February the CDC projected a $48-65 \%$ infection rate and deaths of 0.16 million (with a $0.25 \%$ infection fatality rate) to 1.7 million (1\% fatality rate) over a year starting March 2020 (Fink 2020). Importantly, other empirical studies now point to a case fatality rate close to $1 \%$ (Verity et al. 2020, Mizumoto and Chowell 2020), and other expert estimates suggest a 30-70\% US-wide infection rate (Axelrod 2020, Ramsey 2020). The Ferguson et al. estimates, while slightly more pessimistic, are thus broadly consistent with other projections of COVID-19 transmission.

The mitigation scenario emphasized by Ferguson et al. is a moderate form of social distancing that consists of 7-day isolation for anyone showing coronavirus symptoms, a 14day voluntary quarantine for their entire household, and dramatically reduced social contact for those over 70 years of age. 2 All measures begin in late March. The isolation and household quarantine measures are assumed to be in place for three months and reduced contact for people over 70 lasts four months. Ferguson et al. project that the mitigation scenario will reduce peak hospital demand by two-thirds and total deaths to 1.1 million.

We focus on the mitigation scenario because it approximates what the United States is implementing, albeit unevenly across the country. With perhaps the exception of California, Washington and New York, most US states have not pushed China-style shutdowns of the level necessary to suppress COVID transmission (Glanz et al. 2020). In other words, the US may "flatten the curve" of infection but not stop it entirely. Ferguson et al. also make projections about a "suppression" scenario that includes dramatically reduced contact for the entire population, and involves either a rebound epidemic (that strongly resembles our mitigation scenario) or repeated imposition of social distancing for two years. We view the latter as far from anything being implemented in the United States. One thing to note is that both the no policy and mitigation scenarios only extend through October 1 , so it is reasonable to assume that a vaccine will not be developed in this timeframe. 
A novel feature of our analysis is that we improve upon Ferguson et al.'s estimated mortality projections by accounting for the potential shortages in the supply of hospital intensive care services, for example ICU beds, respirators, and trained staff. Specifically, Ferguson et al.'s headline death projections assume that all COVID-19 patients receive the appropriate medical care, so their projections do not account for potential shortages in ICU beds or respirators. Indeed, it is precisely the possibility of these shortages that account for the policy push to "flatten the curve" and avoid their repercussions. Our approach is to label the Ferguson et al. projections of deaths as "direct deaths" and develop projections of "overflow deaths" which are those that result from hospital ICUs reaching capacity and being unable to serve some COVID-19 patients. As we detail, we project that social distancing would reduce overflow deaths by an additional 630,000 fatalities.

In summary, we project that social distancing reduces COVID-19 caused deaths by 1.76 million deaths. This is composed of reductions of 1.13 million direct deaths and 630,000 overflow deaths. The remainder of this section describes how we develop these projections of the reductions in direct and overflow deaths due to social distancing and their distribution across 9 age categories (Fong et al., 2020).

We begin by reproducing the Ferguson et al. estimates of direct deaths in the US: 2.2 million with no policy and 1.1 million with "mitigation" social distancing. To do so, we develop a method that, under simple assumptions about the progression of coronavirus, allows us to construct the full daily distribution of deaths. This step is necessary because it was infeasible to acquire the full dataset underlying the Ferguson et al. analysis, undoubtedly due to the great demands placed on the authors as they model the progression of COVID-19 and replay updated findings to policymakers.

The approach assumes that daily COVID-19 cases, deaths, and ICU bed demand follow a normal distribution. Normal distributions roughly approximate epidemic growth curves, which are slightly rightskewed since they grow exponentially until reaching herd immunity. Normality is also convenient because given the center (date of peak), height at peak, and width (distance from start to peak), it is possible to recover the full distribution (i.e., daily fatality counts).

The bottom line from this analysis is that social distancing is projected to reduce the number of COVID19 deaths by 1.1 million between March 1 and October 1 . This is simply the difference in the number of direct deaths in the no policy and mitigation scenarios.

\section{CONCLUSIONS}

A large gathering such as the Hajj still holds an increasing risk for future disasters. AS has been presented in this paper with works of several authors and groups, Social Distancing impact on the control of any epidemic or pandemic within mass gathering of the size of the yearly Hajj in Saudi Arabia, can only reduce transmission significantly in the case of known new cases of infection of individuals. It only therefore remains collaboration and continued vigilance in planning efforts remains an integral part of these annual preparations. The development of educational campaigns for pilgrims regarding the possible dangers of this novel pandemic is also crucial. Lessons gained from experiences at the Hajj may also influence planning for mass gatherings of any kind, worldwide. 


\section{REFERENCES}

[1] Ahmed, F., Zviedrite, N., \& Uzicanin, A. (2018). Effectiveness of workplace social distancing measures in reducing influenza transmission: A systematic review. BMC Public Health, 18(1), 518. https://doi.org/10.1186/s12889-018-5446-1

[2] Attanasi, G., Casoria, F., Centorrino, S., \& Urso, G. (2013). Cultural investment, local development and instantaneous social capital: A case study of a gathering festival in the South of Italy. The Journal of Socio-Economics, 47, 228-247. https://doi.org/10.1016/j.socec.2013.05.014

[3] Baum, N. M., Jacobson, P. D., \& Goold, S. D. (2009a). "Listen to the People": Public Deliberation About Social Distancing Measures in a Pandemic. The American Journal of Bioethics, 9(11), 4-14. https://doi.org/10.1080/15265160903197531

[4] Caley, P., Philp, D. J., \& McCracken, K. (2008). Quantifying social distancing arising from pandemic influenza. Journal of the Royal Society Interface, 5(23), 631-639. https://doi.org/10.1098/rsif.2007.1197

[5] Ferguson, N. M., Cummings, D. A. T., Fraser, C., Cajka, J. C., Cooley, P. C., \& Burke, D. S. (2006). Strategies for mitigating an influenza pandemic. Nature, 442(7101), 448-452. https://doi.org/10.1038/nature04795

[6] Fong, M. W., Gao, H., Wong, J. Y., Xiao, J., Shiu, E. Y. C., Ryu, S., \& Cowling, B. J. (2020). Nonpharmaceutical Measures for Pandemic Influenza in Nonhealthcare SettingsSocial Distancing Measures. Emerging Infectious Diseases, 26(5), 976-984. https://doi.org/10.3201/eid2605.190995

[7] Fraser, C., Riley, S., Anderson, R. M., \& Ferguson, N. M. (2004). Factors that make an infectious disease outbreak controllable. Proceedings of the National Academy of Sciences, 101(16), 6146-6151. https://doi.org/10.1073/pnas.0307506101

[8] Greenstone, M., \& Nigam, V. (2020). Does Social Distancing Matter? (SSRN Scholarly Paper ID 3561244). Social Science Research Network. https://doi.org/10.2139/ssrn.3561244

[9] Jones, C., Philippon, T., \& Venkateswaran, V. (2020a). Optimal Mitigation Policies in a Pandemic: Social Distancing and Working from Home (No. w26984; p. w26984). National Bureau of Economic Research. https://doi.org/10.3386/w26984

[10] Jones et al. - 2020-Optimal Mitigation Policies in a Pandemic Social .pdf. (n.d.-a). Retrieved June 11, 2020, from https://www.nber.org/papers/w26984.pdf

[11] Lai, S., Ruktanonchai, N. W., Zhou, L., Prosper, O., Luo, W., Floyd, J. R., Wesolowski, A., Santillana, M., Zhang, C., Du, X., Yu, H., \& Tatem, A. J. (2020a). Effect of nonpharmaceutical interventions for containing the COVID-19 outbreak in China [Preprint]. Infectious Diseases (except HIV/AIDS). https://doi.org/10.1101/2020.03.03.20029843

[12] Markel, H., Lipman, H. B., Navarro, J. A., Sloan, A., Michalsen, J. R., Stern, A. M., \& Cetron, M. S. (2007). Nonpharmaceutical Interventions Implemented by US Cities During the 1918-1919 Influenza Pandemic. JAMA, 298(6), 644.

https://doi.org/10.1001/jama.298.6.644

[13] Peak, C. M., Childs, L. M., Grad, Y. H., \& Buckee, C. O. (2017). Comparing nonpharmaceutical interventions for containing emerging epidemics. Proceedings of the National Academy of Sciences, 114(15), 4023-4028.

https://doi.org/10.1073/pnas.1616438114 
The Need for the Social Distancing on the Crowd Management of Major Events During

Coronavirus: A Case of the Islamic Pilgrim (HAJJ)

[14] Qualls, N., Levitt, A., Kanade, N., Wright-Jegede, N., Dopson, S., Biggerstaff, M., Reed, C., Uzicanin, A., CDC Community Mitigation Guidelines Work Group, Levitt, A., Dopson, S., Frank, M., Holloway, R., Koonin, L., Rasmussen, S., Redd, S., de la Motte Hurst, C., Kanade, N., Qualls, N., Reed, C. (2017). Community Mitigation Guidelines to Prevent Pandemic Influenza-United States, 2017. MMWR. Recommendations and Reports, 66(1), 1-34. https://doi.org/10.15585/mmwr.rr6601a1

[15] Tewari, S., Khan, S., Hopkins, N., Srinivasan, N., \& Reicher, S. (2012b). Participation in Mass Gatherings Can Benefit Well-Being: Longitudinal and Control Data from a North Indian Hindu Pilgrimage Event. PLoS ONE, 7(10). https://doi.org/10.1371/journal.pone.0047291

[16] World Health Organization Writing Group. (2006). Nonpharmaceutical Interventions for Pandemic Influenza, International Measures. Emerging Infectious Diseases, 12(1), 81-87. https://doi.org/10.3201/eid1201.051370

[17] Wu, J. T., Riley, S., Fraser, C., \& Leung, G. M. (2006). Reducing the Impact of the Next Influenza Pandemic Using Household-Based Public Health Interventions. PLoS Medicine, 3(9), e361. https://doi.org/10.1371/journal.pmed.0030361 